\title{
Describing economic agent-based models - Dahlem ABM documentation guidelines
}

\author{
Sarah Wolf ${ }^{\mathrm{a}, *}$, Jean-Philippe Bouchaud ${ }^{\mathrm{b}}$, Federico Cecconic $^{\mathrm{c}}$, Silvano Cincotti ${ }^{\mathrm{d}}$, \\ Herbert Dawid ${ }^{\mathrm{e}}$, Herbert Gintis ${ }^{\mathrm{f}}$, Sander van der Hoog ${ }^{\mathrm{e}}$, Carlo C. Jaeger ${ }^{\mathrm{a}}$, \\ Dmitry V. Kovalevskyg,h, Antoine Mandel ${ }^{\mathrm{i}}$ and Leonidas Paroussos ${ }^{\mathrm{j}}$ \\ ${ }^{a}$ Global Climate Forum \\ E-mail: sarah.wolf@globalclimateforum.org \\ ${ }^{\mathrm{b}}$ Capital Fund Management S.A. \\ ${ }^{\mathrm{c}}$ Laboratory of Agent Based Social Simulation \\ ${ }^{\mathrm{d}}$ Department of Biophysical and Electronic Engineering, University of Genoa \\ ${ }^{\mathrm{e}}$ Fakultät für Wirtschaftswissenschaften, Universität Bielefeld \\ ${ }^{\mathrm{f}}$ Central European University (Budapest) \\ ${ }^{\mathrm{g}}$ Nansen International Environmental and Remote Sensing Centre, St. Petersburg State University, \\ St. Petersburg \\ ${ }^{\mathrm{h}}$ Nansen Environmental and Remote Sensing Centre, Bergen \\ ${ }^{\mathrm{i}}$ Université Paris 1 Panthéon-Sorbonne, Centre d'Économie de la Sorbonne \\ ${ }^{\mathrm{j}} E^{3} M-L a b$, National Technical University of Athens
}

\begin{abstract}
At the 100th Dahlem conference "New Approaches in Economics after the Financial Crisis" a working group devised guidelines for the documentation of computational economic agent-based models, based upon - but differing from - the ODD protocol Grimm et al. (2006, 2010). This paper sketches the motivation for coming up with a new set of guidelines tailored to economic multi-agent modelling, and presents these.

While analytical economic models can often be precisely and concisely stated by a few equations together with an economic interpretation of their elements, a computational agentbased model, as a conceptual piece of work, may not always be a very tangible entity. For example, it is represented by but usually not identical to the (many) equations constituting the computer code. It is therefore not always easy to describe the model in a way that provides the reader with a thorough understanding of the model. The present guidelines are an attempt at standardizing such descriptions to support understanding and communication, as well as the comparability of economic multi-agent models.
\end{abstract}

\section{Introduction}

Complexity economics considers the economy as a complex evolving system, composed of many and heterogeneous agents in interaction. Agent-based models (ABMs) are a tool for studying the system's behaviour at the macro-level as it arises from actions and interactions of many agents. In particular, the focus of this paper is on computational

* Corresponding author.

(c) Baltzer Science Publishers 
ABMs, which implement (generally many) agents at the micro-level on the computer, equipping them with rules for action and interaction. Simulation runs are then used to study the evolution of the system at the macro-level. There is a quickly growing literature on such ABMs in economics, however, an overview over it at the level of generality that would be adapted to this paper is beyond its scope.

By considering trajectories of the system over time, ABMs have a dynamic perspective, that is found wanting in standard equilibrium models which compute an optimal state without considering how society would get to this point. ABM simulations can be seen as a substitute for real-world experiments, which, unlike in physics, cannot be made with the objects of study in economics.

To contrast these advantages of ABMs, there is a disadvantage: the model, as a conceptual piece of work, may be hard to grasp (as further discussed in Section 2). This of course makes describing the model a rather vaguely defined task. In fact, descriptions of (economic) ABMs found in the literature differ widely, and in many cases leave the reader with a sense of "smoke and mirrors" instead of a thorough understanding of the model. This might be one reason why works on ABMs have been rather difficult to publish in many economic journals.

Good descriptions of economic ABMs and their properties are therefore highly desirable. Clearer descriptions would ease the review process of ABMs. This could enhance the facility of publishing ABM-based papers in economic journals. Also, more transparent descriptions would help students learn how to build and analyze ABMs. Finally, similarity in the descriptions of ABMs would make comparison of different $\mathrm{ABMs}$ easier.

A starting point in this direction is to promote standardized descriptions of ABMs. A standard that has been proposed in the field of ecology is the Overview, Design Concepts and Details (ODD) protocol by Grimm et al. (2006, 2010). This paper presents guidelines for describing economic ABMs (in Section 4). In devising these, we considered the ODD protocol as a starting point, but deviated from it where it seemed necessary and useful for the description of socio-economic ABMs (see Section 3).

\section{Computational agent-based models - what exactly is the model?}

Many models used in economics are analytical models, written down in a few equations that come with economic interpretations of the terms occurring in the formulae. The description of the interpretations is mostly done in natural language, possibly using technical terms as well. The equations are concisely and precisely stated in mathematics - a formal language, in which the symbols can be used to perform operations with them. These equations are supposed to describe the behaviour of the system under consideration. Generally, such models are considered clearly defined. A solution to the equations again has an economic interpretation. Solving the equations may require numerical methods, that is, the equations are implemented on a computer and solved 
approximately. The model itself, however, is independent of the details in the computer implementation, and in particular, the solution should be.

(Computational) agent-based models are used to study complex systems precisely because analytical models of these systems are in most cases too difficult to establish. The game of chess provides a summary example: an analytical model of this game not only would be difficult to solve analytically, even writing it down seems incredibly difficult. Implementing the rules according to which the pieces move on a computer is a much more feasible task. It is not so easy to then separate the model from the implementation a priori. However, a computational ABM is not simply identical to the computer code implementing it, as the model should not depend upon each and every implementational detail in the code. One could for example use different formal structures such as lists or arrays to represent the same economic entities. The choice may make a difference for the performance of the code, and the question how long it takes to run a simulation may be very important in a practical sense, but such details should not make a difference for the model itself.

Then what exactly is the model? Generally, there is not a well-defined set of (few) equations together with an economic interpretation, considered to be the model. Rather, the model is a conceptual piece of work that may exist only abstractly - in the head of its developer, for example. Gallegati and Kirman (2012) refer to a " "black box" of computer simulations' used in agent-based computational economics (p. 19). In fact, agents are implemented by a computer program and interact when the program is run. That is, what is represented by a formal language (computer code in this case) are parts of the system under consideration. Describing these does not necessarily provide a complete picture of the model.

And not only is "the scientific conceptual thing" that is the model not always very tangible. Often, it is also not clear from the start what its essential features are. In fact, by considering the overall system behaviour a consequence of many actions and interactions, possibly with many feedback effects that are hard to keep track of, one does not see the overall system behaviour before actually running some simulations. Further, most ABMs have stochastic components, and each run differs from others, so that "the system behaviour" can be studied only with the help of statistical tools. This means that for ABMs, many questions asked by economists cannot be answered a priori, e.g. which effects higher wages will have on employment. Arguably, it is not feasible to require that an ABM be specified explicitly as in a software contract between client and producer of the software before it is built, because the scientist who builds the model starts out with a structure that is known little even to him/herself.

All this of course leads to the question what a good description of an ABM should look like. While mathematics allows precision in scientific communication in many cases, and helps state analytical models unambiguously, it is not the universal remedy for describing ABMs more precisely. Theoretically, it would be possible to reproduce the code using mathematical formulae, but in the vast majority of cases (if not in all of 
them) this endeavour would be utterly useless; such a description would most probably turn out to be unintelligible for a human reader. Some elements may be usefully described using mathematical formulae (such as payoff functions, choice models, learning algorithms) while others, that can be rather easily described in other languages (such as message passing between agents) may be difficult to describe explicitly in mathematics.

A useful mathematical description of a complete ABM would need to be a representation of it, just as the computer code implementing it. As such, it is not uniquely determined. For example, ABMs can be considered dynamical systems because in a simulation, iterations compute one new state after the other from a given initial state of all agents and their environment. However, explicitly writing down the state space and the transition function that define the dynamical system is usually deemed impossible and sometimes unnecessary (Fontan, 2005; Page, 2008). Also, it is not a priori clear what type of a dynamical system should be used. From the point of view of the implementation, an ABM can be considered a deterministic system: when including the seed used by the random number generator in the input to the $\mathrm{ABM}$, the output for a given input is deterministic (e.g. Epstein, 2006, gives a deterministic description of ABMs using a recursive scheme). Hence, randomness can be discussed away with the argument that the computer generates pseudo random numbers. However, ABMs are often considered to be Markov processes ${ }^{1}$ that is, probabilistic systems (Tesfatsion, 2006; Gintis, 2007), and indeed, uncertainty is an essential ingredient for the representation of economic systems. While a mathematics of agent-based models seems a worthy undertaking in itself, it cannot (at this point) offer a description standard for economic ABMs that would provide the reader with a thorough understanding of the complete model.

Summarizing, one can say that despite some works that promote good practice for developing and describing ABMs (e.g. Macal \& North, 2011; Grimm et al., 2006, 2010), there are (as yet) no commonly applied principles how an agent-based economic model should be built or described.

\section{Why a (new) description standard?}

An ABM may be rather easy to explain to non-experts of the field because agents and their interactions, represented in computer code and then simulated by running the program, can be quite an intuitive representation of a real-world economic situation. However, at the level of detail required for scientific communication, the situation is somehow reversed: the ABM might have too many elements to be conveniently communicated in a precise fashion.

A standardization of ABM descriptions for scientific publications is a step towards clarity in communication. This could ease the editorial process for works based on ABMs. ABMs would become more easily comparable, which might help decision

\footnotetext{
${ }^{1}$ See also Gintis and Mandel (2012) on economic ABMs and Markov processes.
} 
makers draw on the results from several modelling approaches when confronted with certain policy questions. Comparability of different economic ABMs would also foster communication and possible collaborations between researchers in this field. The recent financial crisis suggests that novel approaches to modelling the stability and fragility of market systems are likely to be fruitful. New results for a more complete economic theory, obtained from working with ABMs, would probably have a larger impact in the field if presented by a network of cooperating agent-based modellers than if presented by single research groups whose ABM work appears unrelated. Finally, it could be beneficial to economics curricula if the literature offered a larger number of accessible ABMs. Good descriptions that would make these models more easily understandable for students would facilitate teaching a dynamic perspective on economic systems.

Of course, there is not one description of a model for all audiences, for example, a programmer's manual would need to be more detailed than a user's manual. Similarly, different types of language might be most appropriate for model descriptions made for different audiences. A mixture of natural language text (using social science's technical language), mathematical formulae, graphical descriptions, pseudocode, actual code, and models used in computer science to describe certain kinds of computation may be useful. Graphical language seems particularly adapted to give a concise overview of the sequencing occurring in a model, and some agents may be well-described by referring to computational descriptions such as finite state machines. A specification of interaction and communication between parts of the model could be given in first order logic, UML (which may be more intuitive but less clear), or constructive type theory, to provide a choice of examples.

The Dahlem ABM documentation guidelines are proposed as a document for economic journals, that can thus ask authors to provide standardized descriptions of agentbased models. The aim of a description following the guidelines is to provide its reader with a thorough understanding of the model as a conceptual piece of work - the focus is not on the model's implementation. Such a description is not necessarily meant to be published as a paper, but may be included in an (online-) appendix to a paper. In this case, there may be overlap between paper and appendix.

In a model documentation standard, both a common language and a common order in which to describe certain elements of the model are useful, because knowing what to expect generally facilitates understanding, as pointed out by Grimm et al. (2006). Their ODD protocol is a pioneering approach towards standardizing ABM descriptions, rooted in the field of ecology. Anyone who ever had to use a power adapter knows: the nice thing about standards is that there are so many of them. Sticking to the ODD standard however proved difficult for documenting large scale economic agent-based models, for reasons detailed below.

Therefore, at the 100th Dahlem conference "New Approaches in Economics after the Financial Crisis", a working group of about 15 people involved in economic multiagent modelling took the ODD protocol as a starting point in a set of discussions that 
led to the guidelines presented here. Elements that appeared useful also in the economic context were maintained as given in the ODD protocol. For example, the basic structure remains the same: an overview is given, design concepts conveying underlying ideas of the modeller about the model are described, and finally, the detailed information about the model's functional specification is provided. ${ }^{2}$ Hopefully, this facilitates reading economic $\mathrm{ABM}$ descriptions written according to these guidelines also for people who have some experience with descriptions following ODD.

Some elements of the ODD protocol were not taken up at the Dahlem conference, and some new ones were added, to better suit the description of economic ABMs. The structure was slightly rearranged. For example, while the ODD protocol asks for a description of "Process overview and scheduling" in the Overview section already, in the Dahlem guidelines, the Overview section only asks for the kind of activities agents are engaged into. The representation of "Time, activity patterns and activation schemes" is a design concept: the modeller is asked to specify the underlying ideas for this point. However, the actual scheduling may be rather involved in large scale economic ABMs, so that its description is deferred to the Functional Specification section, in order to keep the first two sections concise.

The design concepts themselves were not taken over from the ODD protocol, but the group came up with design concepts directly geared towards economic ABMs. Some design concepts from the ODD protocol recur in these guidelines, e.g. "Learning", or are rephrased, such as "Prediction" that has been changed to "Forecasting", which is the more usual term in the case of economic agents. Others are new, such as "Interaction protocols and information flows" - while ODD's design concepts focus on the agents, an additional focus on the design of the interaction between agents was desired for describing economic models.

Apart from the context change, two main issues implied deviations from the ODD protocol. First, ODD seems appropriate for rather small models, for example in its aim to enable the reader of a description to reimplement the model that was described. Economic ABMs can be rather large, especially when modelling a whole economy, and not just a single market (see for example the EURACE model (Dawid et al., 2011), or the Lagom models (Mandel et al., 2009; Wolf et al., 2012)). A description of a Lagom model that strictly follows ODD would easily fill 50-80 pages. It would certainly be too much to ask a reviewer of a paper, which presents results based on the model, to first read this much in order to obtain an understanding of the model. That is, the purpose of ODD (enabling a reimplementation of the model) was simply not feasible for the models considered in the economic context. With this change of purpose, also the protocol needed to be changed.

\footnotetext{
${ }^{2}$ This may lead to some doubling of information between the Design Concepts and the Functional Specification section. However, the first two sections may be seen as a document for reading page by page, while the third section rather presents a work of reference. Hence, this potential doubling of information is accepted.
} 
Secondly, ODD seems geared toward single applications of models. This may be related to the first point, in that a small model may be built to answer a specific research question from the outset. Economic ABMs, and especially the large-scale models, are often built without a strict focus on a single research question. The role of such models can be compared to the role of a city map, which is a model of the city, after all. A map is not usually produced in order to answer a specific question, but rather to answer questions of the type "how do I get from A to B?". Similarly, an economic ABM may be built as a representation of the economy, to answer questions of the type "which consequences can some action by the government/the central bank etc. have?" ODD's focus on a single purpose of the model, as well as on a specific initialization used, therefore is not so useful in dealing with (large-scale) economic ABMs. In contrast, the Dahlem guidelines draw a clear distinction between the description of the model and descriptions of simulations done with it. To provide an example, consider the initialization of an ABM. ODD asks for the numbers of agents and their state variables at initialization of the model, while the Dahlem guidelines ask which input is required and how the initial state of the model is obtained using this input. That is, in order to describe the model per se, one may say "the number of firms is set by the model user" instead of having to refer to a specific simulation with its number of firms. Simulations done with the model, data used in simulations, and statistical tools used to analyze the model behaviour (e.g. characteristics of a Monte-Carlo procedure, such as how many iterations, how many time steps per iteration, whether the seed is random or not, etc.) should of course also be described in a systematic and precise fashion, however, structuring these tasks goes beyond the present guidelines.

\section{The Dahlem ABM documentation guidelines}

The authors should provide a description of their model using the following template, consisting of overview, design concepts, and functional specification. A summary template is provided in Table 1; details, that is, questions to be answered for the respective points, are presented below. Note that the description concerns the model per se; specific research questions and computational experiments are not considered here. Modellers are encouraged to make source code and data publicly available. If this is not possible, it should be stated.

\subsection{Overview (max. 3 pages)}

This section gives an overview of the model in natural language (with embedded technical concepts). Authors are encouraged to illustrate it by an image they find appropriate. This overview should fit into the following structure.

- Rationale

What is the object under consideration (e.g. a financial market, the world economy)? What is the intended usage of the model (e.g., theory generation, forecast- 
Table 1

Dahlem guidelines summary template.

\begin{tabular}{ll}
\hline Overview & Rationale \\
(max. 3 pages) & Agents \\
& Other entities \\
& Boundaries \\
& Relations \\
& Activities \\
& Time, activity patterns and activation schemes \\
Design Concepts & Interaction protocols and information flows \\
(max. 3 pages) & Forecasting \\
& Behavioural Assumptions and Decision Making \\
& Learning \\
& Population Demography \\
& Levels of Randomness \\
& Miscellaneous \\
& Description of Agents and Other Entities, action and interaction \\
Functional Specification & Initialization \\
& Run-time input \\
\hline
\end{tabular}

ing, policy analysis, etc.)? Which issues can be investigated (e.g. determinants of wages, evolution of trade networks)?

- Agents

What kind of agents (decision-making entities) are considered in the model? Is there a refined taxonomy of agents? In particular, are there agent groupings which are considered relevant?

- Other entities

What are the other entities which are time-evolving but not decision-making?

- Boundaries

What are additional inputs to the model at runtime? Which outside influences on the model are hence represented?

- Relations

What kind of relationships structure the agents' interactions (e.g. networks)? To which extent do these represent institutions (e.g. labor market, states)?

- Activities

What kind of actions and interactions are the agents engaged into?

\section{2. $\quad$ Design concepts (max. 3 pages)}

In this part of the documentation, various aspects of the general modelling approach should be spelled out using natural language. Details of how the actual model looks like should be provided in the following section 'Functional Specification'. 
- Time, activity patterns and activation schemes What is the basic sequence of events in the model? Are activities by agents triggered by a central clock or by actions, respectively messages sent, by other agents? What is the interpretation of one time unit in the model?

- Interaction protocols and information flows What are the general properties of the protocols governing the interaction between agents? How is determined which agents can interact with each other (e.g. all agents, local interaction, networks, ...)? What kind of information is available to each agent? If agents interact within institutional frameworks like firms or markets, what are the main properties of these institutions (e.g. auction markets, matching markets, ...)?

- Forecasting Are agents in the model forward looking or purely backward looking? If agents are forward looking, what is the basic approach to modelling forecasting behaviour (e.g. naive forecasting, econometric methods, CI-methods, ...)?

- Behavioural Assumptions and Decision Making Based on which general concepts is decision making behaviour of the different types of agents modelled (e.g. based on experimental evidence, solution of some optimization problem, heuristics, documented behaviour of real world firms, established models from the literature, ...)? If the decision making of certain agents is influenced by their beliefs, how are these beliefs formed?

- Learning Are decision rules of agents changed over time? If yes, which types of algorithms are used to do this?

- Population Demography Can agents drop out of the population and new agents enter the population during a simulation run? If yes, how are exit and entry triggered?

- Levels of Randomness

How do random events and random attributes affect the model?

- Miscellaneous

Any important aspects of the used modelling approach that do not fit any of the items above should be explained here. For example, mathematical properties of the model that are considered relevant should be stated here.

\subsection{Functional specification}

This section provides a detailed description of the agents, other entities, their actions and interactions, the initialization procedure, and runtime-input requirements. It is closer to the implementation of the model, but need not be so detailed as to allow the reader to re-implement it. Rather, the level of detail should be determined by what is needed to provide a good understanding while not overly straining the reader's patience. Using natural language and pseudocode is encouraged. 
Table 2

Extract from a possible table of firms' state variables. Here, "Region" is an object in the model, $N_{G}$ denotes the number of goods (sectors) in the model, $w$ the periodicity of the step that can change the circulating input coefficients of a firm, "volatile" means that a variable can change more than once per period. Any useful categories can be used in the last two columns, given that they are also explained.

\begin{tabular}{lllll}
\hline Name & Type & Description & Updating & Initialization \\
\hline Firm & & & & \\
\hline region & Region & $\begin{array}{l}\text { Region where the Firm is } \\
\text { located }\end{array}$ & fixed & computed \\
circulatingInputCoefficients & {$[0,1]^{N_{G}}$} & $\begin{array}{l}\text { Input coefficients for circu- } \\
\text { lating capital }\end{array}$ & periodicity $w$ & computed \\
$\begin{array}{l}\text { producedQuantity } \\
\text { wage }\end{array}$ & $\mathbb{R}_{+}$ & $\begin{array}{l}\text { Quantity of good produced } \\
\text { Wage offered in new work } \\
\text { contracts }\end{array}$ & $\begin{array}{l}\text { periodicity } 1 \\
\text { volatile }\end{array}$ & $\begin{array}{l}0 \\
\text { user input }\end{array}$ \\
\hline
\end{tabular}

- Description of Agents and Other Entities, action and interaction What, in detail, are the Agents and Other Entities in the model? What Agent/Other Entity does what and in which order? For each kind of Agents/Other Entities, what are the model state variables (in principle what is needed to restart a simulation, i.e., all auxiliary variables that can be computed from these do not need to be listed) and parameters? List their type, that is, dimensions and admissible range, a short description of what they represent, units of measurement, how often they are updated (in models with different time scales) and how they are initialized, e.g., as a fixed value, from user-given input data or by a computation out of these data. Table 2 provides an example, further columns can be added if other information seems useful. Depending on the length, the table should be put into an appendix. What information and with whom does each kind of agent exchange for decisionmaking? When are state variables updated? How are state variables updated (specify equations, diagrams, or pseudocode for algorithms related to rules-of-thumb, learning, adaptation, forecasting, interaction, etc.)?

- Initialization How is the model initialized? Which kind of input is needed? How is the initial state obtained from the input? Are the initial values chosen arbitrarily or based on data? In the latter case, what kind of data is needed?

- Run-time input

Does the model use input from external sources that drive the model " drive" means that one or more state variables or processes are affected by how these external variables change over time, but these external variables are not themselves affected by the internal variables of the model)? Are there data files or other models that represent these external processes? If so, what kind of data is required to feed the model at runtime, (e.g. time series of the oil price or temperature data)? Include, if possible, references to relevant literature, or a description of the external models. If a model does not use external data, please state this here. 


\section{Conclusion and outlook}

This paper has introduced the Dahlem ABM documentation guidelines, developed at the 100th Dahlem conference in order to encourage standardized descriptions of economic ABMs. It also discussed the motivation for proposing these guidelines and for deviating from the ODD protocol, on which the guidelines are based. Since an ABM, as a as conceptual piece of work, is rather vaguely defined, a good description is highly desirable to make it accessible for scientific communication. Setting a description standard is a step towards improving ABM descriptions.

For the moment, the guidelines are not set in stone, but should be considered an evolving document. Of course, a changing standard is somewhat self-contradictory, however, the developers of the guidelines are aware that the guidelines should be tested; the need to make some modifications may arise when using them. Therefore, a first step towards a quality control of the guidelines themselves is the task of applying them to describe ABMs. A first description following the guidelines can be found in (Wolf et al., 2012). While this test showed that (at least for the Lagom model described in this case) the guidelines lead to minor redundancies, the general impression was that following the guidelines is feasible.

Agent-based modellers from the field of economics are encouraged to try the guidelines as well. Comments to the corresponding author on how to improve them are highly appreciated. Complexity Economics encourages submissions of ABM papers that provide a description according to these guidelines. Having made some experience, an improved version of the guidelines is then supposed to be fixed as the actual documentation standard and circulated to economic journals.

\section{References}

Dawid, H., Gemkow, S., Harting, P., van der Hoog, S., \& Neugart, M. (2011).The Eurace@ Unibi Model: An Agent-Based Macroeconomic Model for Economic Policy Analysis. Working Paper, Bielefeld University.

Epstein, J. M. (2006). Remarks on the foundations of agent-based generative social science. In Tesfatsion, L. and Judd, K. (Eds.), Agent-Based Computational Economics. Elsevier, North-Holland.

Fontana, M. (2005). Computer Simulations, Mathematics and Economics. Working Paper No 06/2005. Dipartimento di Economia "S. Cognetti de Martiis."

Gallegati, M. \& Kirman, A. (2012). Reconstructing economics: Agent-based models and complexity. Complexity Economics, 1, 5-31. doi: 10.7564/12-COEC2.

Gintis, H. (2007). The dynamics of general equilibrium. Economic Journal, 117(523), 1280-1309. doi: 10.1111/j.1468-0297.2007.02083.x

Gintis, H. \& Mandel, A. (2012). The stability of Walrasian general equilibrium. http://hal-paris1.archivesouvertes.fr/halshs-00748215.

Grimm, V., Berger, U., Bastiansen, F., Eliassen, S., Ginot, V., Giske, J., Goss-Custard, J., Grand, T., Heinz, S., Huse, G., Huth, A., Jepsen, J. U., Jørgensen, C., Mooij, W. M., Müller, B., Pe'er, G., Piou, C., Railsback, S. F., Robbins, A. M., Robbins, M. M., Rossmanith, E., Rüger, N., Strand, E., Souissi, S., Stillman, R. A., Vabø, R., Visser, U., \& DeAngelis, D. L. (2006). A standard protocol for describing individual-based and agent-based models. Ecological Modelling, 198, 115-126. doi: 10.1016/j.ecolmodel.2006.04.023. 
Grimm, V., Berger, U., De Angelis, D. L., Polhill, J. G., Giske, J., \& Railsback, S. F. (2010). The ODD protocol: A review and first update. Ecological Modelling, 221, 2760-2768. doi: 10.1016/ j.ecolmodel.2010.08.019

Macal, C. M. \& North, M. J. (2011). Introductory tutorial: Agent-based modeling and simulation. In Jain, S., Creasey, R., Himmelspach, J., White, K., and Fu, M. (Eds.), Proceedings of the 2011 Winter Simulation Conference (pp. 1456-1469).

Mandel, A., Fürst, S., Lass, W., Meissner, F., \& Jaeger, C. C. (2009). Lagom generiC: An agent-based model of growing economies. ECF Working Paper, 1.

Page, S. E. (2008). Agent-based models. In Durlauf, S. N. and Blume, L. E. (Eds.), The New Palgrave Dictionary of Economics (pp. 47-51). Palgrave Macmillan. doi: 10.1057/9780230226203.0016.

Tesfatsion, L. (2006). Agent-based computational economics: A constructive approach to economic theory. In Tesfatsion, L. and Judd, K. (Eds.), Agent-Based Computational Economics. Elsevier, North-Holland.

Wolf, S., Fürst, S., Mandel, A., Lass, W., Lincke, D., Pablo-Martì, F., \& Jaeger, C. (2012). A multiagent model of several economic regions. Environmental Modeling and Software. doi: 10.1016/ j.envsoft.2012.12.012 